\title{
Etude théorique et expérimentale de l'interaction entre une stratification et un écoulement de recirculation dans une cavité
}

\author{
par D. Grand, A. Latrobe, R. Martin \\ et Ph. Vernier \\ Service des Transierts Thermiques - Centre d'Etudes Nucléaires de Grenoble \\ B.P. 85 Centre de tri, 38041 Grenoble Cedex
}

\section{1 - Introduction}

Dans la réalisation dite intégrée dont le type est Phénix, la thermohydraulique de l'intercuve des réacteurs à neutrons rapides pose des problèmes où hydrodynamique et thermocinétique sont couplées. On peut dénombrer trois catégories de phénomènes.

Tout d'abord, des phénomènes de convection forcée existent à cause des jets de sodium qui sortent des échangeurs. Ensuite, des 'phénomènes de convection naturelle peuvent se développer le long des parois verticales. Enfin, des phénomènes de stratification sont induits par les parois supérieures plus chaudes que le fluide ambiant et presque horizontales; les grandes dimensions de l'intercuve accentueront la stratification de la masse du fiuide, tandis que les effets thermoconvectifs seront confinés au voisinage des parois.

Une des questions posées par le projeteur concerne la répartition des températures sur la paroi extérieure, quasiadiabatique, de l'intercuve, celle-ci pouvant être le siège de contraintes thermiques dangereuses. Une autre question concerne la chaleur totale qui transite par l'intercuve, by-passant l'échangeur et influençant par là-même le niveau absolu de température du réacteur.

Cette communication présente une étude de la convection mixte dans une cavité rectangulaire en vue d'essayer de simuler certains de ces phénomènes.

L'écoulement dans une cavité a été l'objet d'études de Batchelor [1] et Squire [2] qui ont démontré que, pour une cavité circulaire et dans la limite des grands nombres de Reynolds, l'écoulement se réduisait à un noyau à vorticité constante se raccordant par une couche limite aux parois solides. En présence de variations de température aux parois, une température constante s'établit dans le noyau (Grinshaw) [3].
Burggraf [4] a tenté d'appliquer ces résultats au cas d'une cavité rectangulaire, dans l'hypothèse de l'existence d'un tourbillon unique dans l'ensemble de la cavité. Nous verrons que, non seulement la géométrie rectangulaire, mais la présence de forces de volume, limitent les possibilités de cette démarche: plusieurs tourbillons peuvent apparaître dans la cavité.

Nous présentons, au paragraphe 2, des résultats théoriques obtenus par résolution numérique des équations de Navier-Stokes. Nous présentons ensuite les résultats expérimentaux fournis par une installation décrite au paragraphe 3 .

\section{2 - Etudes théoriques}

\section{1 - Formulation du problème}

Le modèle physique est représenté sur la figure 1 , soit une cavité rectangulaire de largeur $d$, et de hauteur $h$. Le côté horizontal inférieur est animé d'une vitesse de translation $V$. Les autres côtés sont immobiles par rapport aux axes de référence. Les parois horizontales sont à température constante $T_{0}$ et $T_{1}$, la température $T_{1}$ de la paroi supérieure étant, dans tous les cas étudiés, la plus élevée de façon à ce qu'une stratification stable soit établie. Les parois verticales sont athermanes.

Le mode d'entraînement du fluide par une paroi mobile, utilisé dans ce modèle, diffère de ce qu'il est dans la réalisation expérimentale. Dans celle-ci, en effet, c'est l'écoulement dans un canal qui produit la recirculation dans la cavité. Plusieurs raisons nous ont amené à nous éloigner ainsi de l'expérience. En premier lieu, l'entraîne- 
ment dans la couche de mélange est marqué, pour la plupart des essais expérimentaux, par des phénomènes de turbulence libre qui ne sont pas pris en compte dans le présent calcul, où l'on suppose l'écoulement laminaire. En deuxième lieu, c'est principalement l'influence de la recirculation sur la stratification qui nous intéresse, et non son mode de génération que l'on présume secondaire.

Par ailleurs le choix d'une paroi défilante, évite la prise en compte de points singuliers difficiles à traiter numériquement.

Les hypothèses suivantes sont faites quant à la nature du fluide :

- incompressible et newtonien;

- les propriétés physiques sont indépendantes de la température, à l'exception des variations de densité dans le terme de forces de volume (hypothèse de Boussinesq).

$\mathrm{Si}$ on suppose l'écoulement laminaire, les équations de conservation pour la vorticité et la température avec l'intermédiaire de la fonction de courant, s'écrivent:

$$
\begin{aligned}
& \frac{\partial}{\partial x}\left(\frac{\partial \psi}{\partial y} \cdot \Omega\right)-\frac{\partial}{\partial y}\left(\frac{\partial \psi}{\partial x} \Omega\right) \\
&= \frac{1}{\operatorname{Re}}\left(\frac{\partial^{2} \Omega}{\partial x^{2}}+\frac{\partial^{2} \Omega}{\partial y^{2}}\right)+\frac{\mathrm{Gr}}{\operatorname{Re}^{2}} \frac{\partial T}{\partial x} \\
& \frac{\partial}{\partial x}\left(\frac{\partial \psi}{\partial y} T\right)-\frac{\partial}{\partial y}\left(\frac{\partial \psi}{\partial x} T\right)=\frac{1}{\operatorname{Re} \cdot \operatorname{Pr}}\left(\frac{\partial^{2} T}{\partial x^{2}}+\frac{\partial^{2} T}{\partial y^{2}}\right) \\
& \frac{\partial^{2} \psi}{\partial x^{2}}+\frac{\partial^{2} \psi}{\partial y^{2}}=-\Omega
\end{aligned}
$$

La vorticité $\Omega$ et la fonction de courant $\psi$ sont reliées aux composantes de la vitesse par les relations de définition :

$$
u=\frac{\partial \psi}{\partial y}
$$

$$
\begin{gathered}
v=-\frac{\partial \psi}{\partial x} \\
\Omega=\frac{\partial u}{\partial y}-\frac{\partial v}{\partial x}
\end{gathered}
$$

Il est à remarquer, que l'équation (3) nécessaire à la fermeture du système (il y a trois variables dépendantes $\psi, \Omega, 7$ ) résulte des relations de définition (4), (5), (6).

Les équations sont écrites sous forme adimensionnelle, les grandeurs de référence étant: la largeur $d$ de la cavité, la vitesse de translation $U$ et $\Delta T=T_{1}-T_{0}$, l'écart de température entre les faces horizontales.

Trois nombres ou groupements adimensionnels apparaissent; le nombre de Reynolds Re, le nombre de Péclet Re.Pr, le paramètre de la convection mixte $G r / \operatorname{Re}^{2}$ :

$$
\operatorname{Re}=\frac{U d}{\nu} ; \frac{\mathrm{Gr}}{\operatorname{Re}^{2}}=\frac{g \beta \Delta T d}{U^{2}} ; \operatorname{Pr}=\frac{\nu}{\alpha}
$$

Les conditions d'imperméabilité des parois, et d'adhérence du fluide à celles-ci conduisent aux conditions aux limites suivantes pour la fonction de courant:

$$
\Psi=0 \text { sur toute la frontière du domaine }
$$

$\frac{\partial \psi}{\partial n}=0$ sur les parois immobiles

$$
\frac{\partial \psi}{\partial n}=1 \text { sur la paroi mobile }
$$

Pour la température;

$$
\begin{aligned}
T & =0 \text { et } T=1 \text { sur les parois horizontales } \\
\text { inférieure et supérieure } & \\
\frac{\partial T}{\partial n} & =0 \text { sur les côtés verticaux }
\end{aligned}
$$

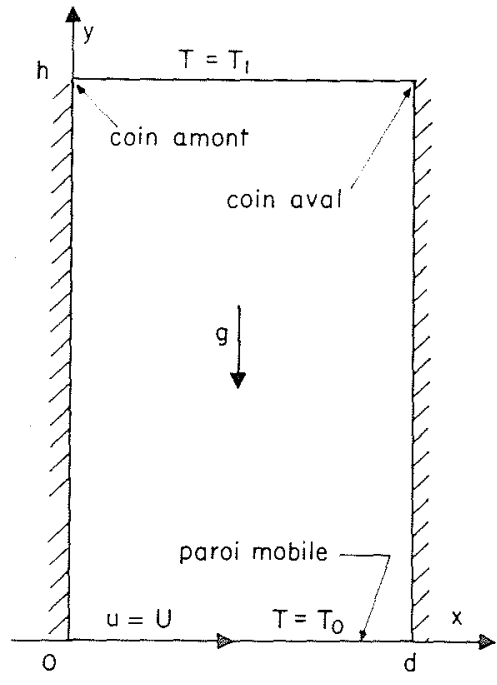

1/ Modèle physique.

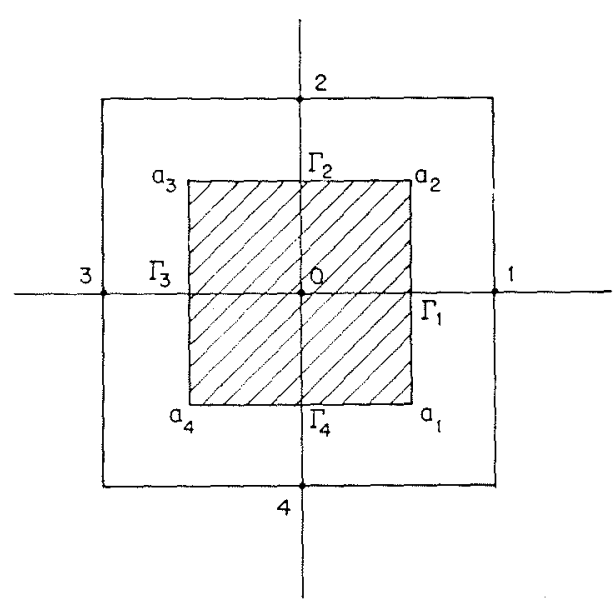




\section{2 - Méthode de résolution numérique}

Le système d'équations aux dérivées partielles (1) à (3) est résolu par une méthode aux différences finies suivant un schéma développé par Gosmann et al. [5], qui présente deux caractéristiques importantes:

- sa forme conservative;

- la discrétisation des termes convectifs.

\section{a) Caractère conservatif des équations discrètes}

Ce n'est pas à partir des équations locales, mais de leur forme intégrale, qu'est construit le système d'équations algébriques associé.

Le domaine plan étant recouvert d'un réseau maillé orthogonal, soit $O$ un nœud courant $(I=1,2,3,4$ les points voisins) et $B$ la maille centrée sur $O$. Les équations locales sont intégrées sur l'aire $B$. A titre d'exemple, l'équation de transport de la vorticité devient (fig. 2) :

$\iint_{B} \operatorname{div} \vec{u} \Omega d B-\frac{1}{\operatorname{Re}} \iint_{B} \operatorname{div} \overrightarrow{\operatorname{grad}} \Omega d B$

$$
=\frac{\mathrm{Gr}}{\operatorname{Re}^{2}} \iint_{B} \frac{\partial T}{\partial x} d B
$$

L'emploi du théorème de la divergence permet de transformer les intégrales de surface du membre de gauche en intégrales de contour. Si $\Gamma$ est la frontière de $B$ et $\vec{n}$ la normale à $\Gamma$ dirigée vers l'extérieur de $B,(12)$ peut être récrite :

$$
\begin{aligned}
\oint_{\Gamma} \vec{u} \Omega \cdot \vec{n} d \Gamma-\frac{1}{\operatorname{Re}} \oint_{\Gamma} \overrightarrow{\operatorname{gra} d} \Omega \cdot \vec{n} d \Gamma \\
=\frac{\mathrm{Gr}}{\operatorname{Re}^{2}} \iint_{B} \frac{\partial T}{\partial x} d B
\end{aligned}
$$

La frontière $\Gamma$ est un quadrilatère de côtés parallèles aux axes; soit $\Gamma_{i}$ le côté qui est compris entre le point courant et le point voisin $I$. Grâce à cette propriété du contour d'intégration, l'équation (13) prend la forme:

$$
\begin{aligned}
\sum_{i=1}^{4}\left(\int_{\Gamma_{i}} \vec{u} \Omega \cdot \vec{n} d \Gamma\right. & \left.-\frac{1}{\operatorname{Re}} \int_{\Gamma_{i}} \overrightarrow{\operatorname{gra} d} \Omega \cdot \vec{n} d \Gamma\right) \\
= & \frac{\mathrm{Gr}}{\operatorname{Re}^{2}} \iint_{B} \frac{\partial T}{\partial x} d B
\end{aligned}
$$

La discrétisation consiste à évaluer les intégrales linéiques qui apparaissent dans cette équation grâce au théorème de la moyenne:

$$
\begin{aligned}
& \sum_{i=1}^{4}\left(\bar{\Omega}_{\Gamma_{i}} \int_{\Gamma_{i}} \vec{u} \cdot \vec{n} d \Gamma-\frac{1}{\operatorname{Re}} \overrightarrow{(\overrightarrow{\operatorname{gad}} \Omega \cdot \vec{n})_{\Gamma_{i}}} \int_{\Gamma_{i}} d \Gamma_{i}\right) \\
& =\frac{\mathrm{Gr}}{\operatorname{Re}^{2}} \iint_{B} \frac{\partial T}{\partial x} d B
\end{aligned}
$$

Dans cette expression $\bar{\Omega}_{\Gamma_{i}}$ et $\overline{(\overrightarrow{\operatorname{grad}} \Omega \cdot \vec{n})} \Gamma_{1}$ sont des valeurs moyennes sur le côté $\Gamma_{i}$.
$\int_{\Gamma_{i}} \vec{u} \cdot \vec{n} \cdot d \Gamma$ est le débit volumique qui sort par la face $\Gamma_{i}$ de la frontière. Cette quantité peut être exprimée en terme des valeurs prises par la fonction courant sur les nœuds du maillage.

\section{b) Discrétisation des termes convectif́s (schéma amont.)}

La discrétisation du membre de gauche de l'équation (15) est complétée par le choix des valeurs moyennes.

Le gradient apparaissant dans le terme de diffusion est approché à l'aide d'une différence centrée.

L'estimation de la valeur moyenne prise par la vorticité sur le côté $\Gamma_{i}$ du contour présente une difficulté plus grande. La caractéristique du schéma aux différences dit amont, est de lier le choix de cette valeur à la direction de l'écoulement :

- si $\int \vec{u} \cdot \vec{n} d \Gamma$ est positif, c'est-à-dire si l'écoulement sort du domaine $B$ par la face $\Gamma_{i}$, on prend:

$$
\overline{\Omega_{\Gamma_{i}}}=\Omega_{0}
$$

$-\operatorname{si} \int_{\Gamma_{\mathfrak{t}}} \vec{u} \cdot \vec{n} d \Gamma$ est négatif,

$$
\overline{\Omega \Gamma}_{i}=\Omega_{i}
$$

Ce choix ne fait que respecter le caractère hyperbolique des termes de convection.

A ce stade, nous disposons d'un système d'équations algébriques susceptible d'un traitement numérique. La méthode de résolution employée est une méthode de point fixe avec relaxation.

Un maillage à pas variable est utilisé; il est resséré au voisinage des parois. Pour les résultats présentés, le réseau comptait dans les cas extrêmes $31 \times 31=961$ nœuds.

Le schéma conservatif n'est pas seulement préconisé parce que l'ingénieur pourra constater qu'il permet un bilan physique des grandeurs transportées sur chaque maille $\mathbf{B}$. Il fournit dans la plupart des cas une précision de $O\left(h^{2}\right)$ si $h$ est la dimension des pas du maillage. Ce résultat est bien connu pour les termes diffusifs de (13). On montre [10] que le schéma conservatif amont des termes convectifs de (13) a une erreur de troncature telle que :

$$
\begin{aligned}
\int_{\Gamma 1+\Gamma 3} \vec{u} \Omega \cdot \vec{n} d \Gamma & =\text { «schéma amont » } \\
& +1 / 2\left(h_{1}-h_{3}\right)|u| \frac{\partial \Omega}{\partial x} \int_{\Gamma_{i}} d \Gamma \\
& +O\left(h^{2}\right)
\end{aligned}
$$

Le schéma est rigoureusement d'ordre 2 lorsque les pas sont constants $\left(h_{1}=h_{3}\right)$. On peut donc dire qu'on évitera le phénomène de viscosité numérique $\nu_{N}=+1 / 2 h|u|$ lorsque les pas $h_{i}$ seront convenablement choisis. Enfin il faut souligner la stabilité inconditionnelle du schéma amont alors que le schéma centré d'ordre 2, conservatif ou non, est généralement instable. 


\section{3 - Résultats}

2.3.1 - Un premier résultat intéressant est l'évolution de la structure de l'écoulement avec le seul paramètre de la convection mixte $\mathrm{Gr} / \operatorname{Re}^{2}$. La figure 3 présente le tracé des lignes de courant pour des essais ayant en commun une valeur faible du nombre de Reynolds, $R e=100$. Pour trois des cas, ( 3 A, B, C) le nombre de Prandtl voisin de l'unité correspond à un fluide classique; dans le dernier (3 D) il a une valeur caractéristique d'un métal liquide.

Lorsque le groupement $\mathrm{Gr} / \mathrm{Re}^{2}$ est nul, le champ de l'écoulement n'est pas affecté par la distribution des températures. Un tourbillon principal se développe dans la plus grande partie de la cavité, et deux tourbillons secondaires de très faible importance apparaissent dans les coins de la cavité opposés à la paroi mobile. La rotation de ces derniers est dans le sens négatif, cependant que celle du tourbillon principal a lieu dans le sens trigonométrique. Ce résultat corrobore ce qui fut précédemment obtenu pour l'écoulement isotherme [6] [7].

Pour $\mathrm{Gr} / \mathrm{Re}^{2}=0,4$ le tourbillon secondaire du coin amont (fig. $3 \mathrm{~B}$ ) s'est nettement développé, cependant que celui du coin aval a disparu.

Pour une valeur supérieure du groupement $\mathrm{Gr} / \mathrm{Re}^{2}=2$, l'extension du tourbillon secondaire s'est poursuivie jusqu'à ce que celui-ci occupe toute la partie supérieure de la cavité (fig. $3 \mathrm{C}$ ).

De par la stratification stable, une particule fluide froide, convectée par le tourbillon principal, présente un défaut de poussée lorsqu'elle passe en partie haute chaude de la cavité. Elle est soumise à une force de rappel vers le bas. Cet effet est responsable de la diminution du tourbillon principal lorsqu'on augmente l'importance relative des forces de volume, dont l'ordre de grandeur est donné par $\mathrm{Gr} \cdot \mathrm{Re}^{-2}$.

La structure de l'écoulement est fortement influencée également par l'importance de la conductivité thermique du fluide. Le dernier cas présenté (fig. $3 \mathrm{D}$ ), montre que pour un fluide plus conducteur, l'influence de la stratification sur la structure de l'écoulement est diminuée.

II est en effet néceșsaire pour obtenir une structure d'écoulement proche de celle représentée dans la figure $3 \mathrm{C}$ de prendre une valeur dix fois supérieure du paramètre de convection mixte. Toutefois si les structures de l'écoulement sont semblables, les champs de température sont très différents (fig. $3 \mathrm{E}$ et $3 \mathrm{~F}$ ).

2.3.2 - L'évolution du champ de température avec le nombre de Reynolds est représentée à la figure 4 , pour une une valeur nulle du paramètre de la convection mixte $\left(\mathrm{Gr} \cdot \mathrm{Re}^{-2}=0\right)$. Avec des valeurs croissantes du nombre de Reynolds, la température tend à devenir uniforme et voisine de celle de la paroi mobile dans une grande partie de l'écoulement. Les isothermes vont en se resserrant sous la paroi chaude (fig. $4 \mathrm{~A}, \mathrm{~B}, \mathrm{C}$ ).

Ce résultat confirme l'analyse de Prandtl [8]. Selon celleci pour des fluides classiques et dans la limite des grands nombres de Reynolds, la température (ainsi que la vorticité) est uniforme dans le noyau de la recirculation.

Par contre, pour un fluide très conducteur $\left(\operatorname{Pr}=10^{-2}\right)$, l'influence des effets convectifs sur le champ de température est considérablement diminuée. La stratification n'est rom- pue que pour de grands nombres de Reynolds (fig. 4 D). Bien qu'obtenus pour le même nombre de Péclet égal à 350 , les isothermes $4 \mathrm{~A}$ et $4 \mathrm{D}$ présentent des différences notables.

2.3.3 - Le transfert de chaleur dans la cavité carrée est analysé maintenant. La figure 5 regroupe l'ensemble des essais numériques réalisés. Le nombre de Nusselt moyen y est porté en fonction du nombre de Reynolds; les courbes obtenues sont paramétrées avec le groupement $\mathrm{Gr} / \mathrm{Re}^{2}$. Pour ces essais, le nombre de Prandtl est égal à 3.5. Les essais réalisés pour un métal liquide $\operatorname{Pr}=10^{-2}$ ont montré que, pour la gamme de Reynolds considérée, le nombre de Nusselt différait très peu de l'unité, valeur correspondant à un régime de conduction pure.

L'évolution du nombre de Nusselt moyen est marquée par:

- une croissance avec le nombre de Reynolds, le paramètre de la convection mixte étant maintenu constant;

- une décroissance avec le paramètre de la convection mixte, à nombre de Reynolds fixé.

Lorsque les forces de volume sont négligées

$$
\left(\mathrm{Gr} / \mathrm{Re}^{2}=0\right) \text {, }
$$

les valeurs du nombre de Nusselt sont corrélées par l'équation :

$$
\overline{\mathrm{N} u}=0,380 \operatorname{Re}^{0.5}
$$

\section{3 - Etudes expérimentales}

\section{1 - Dispositif expérimental}

Le dispositif expérimental consiste essentiellement en une cavité cubique $(9 \mathrm{~cm}$ d'arête) en acier inoxydable, taillée dans la masse débouchant à la partie inférieure sur un canal horizontal de section rectangulaire (1) (cf. fig. 6), de longueur suffisante pour que le régime hydraulique soit établi. La face supérieure de la cavité est chauffée indirectement par effet Joule, les deux faces verticales normales à l'écoulement (3) peuvent être considérées comme athermanes. Deux hublots de verre de qualité strioscopie constituent les deux autres faces verticales (4)

Dans la cavité, les distributions de température sont relevées dans le plan vertical de symétrie (5) au moyen de thermocouples disposés régulièrement sur un support horizontal (6), pouvant être déplacé verticalement.

Le relevé de température est complété par des visualisations des gradients thermiques dans la cavité par la strioscopie en couleurs. Cette méthode, précédemment utilisée au Service des Transferts Thermiques, est fondée sur la déviation d'un rayon lumineux par un milieu transparent présentant des variations de l'indice de réfraction [10] [11].

La figure 7 donne le schéma de principe du montage optique, dit aller-retour, que nous avons adopté pour son encombrement réduit. Après une première traversée de la section d'essais, le faisceau lumineux est réfléchi par un miroir concave. Il traverse une seconde fois la section d'essais et est focalisé. Dans le plan de focalisation, on 


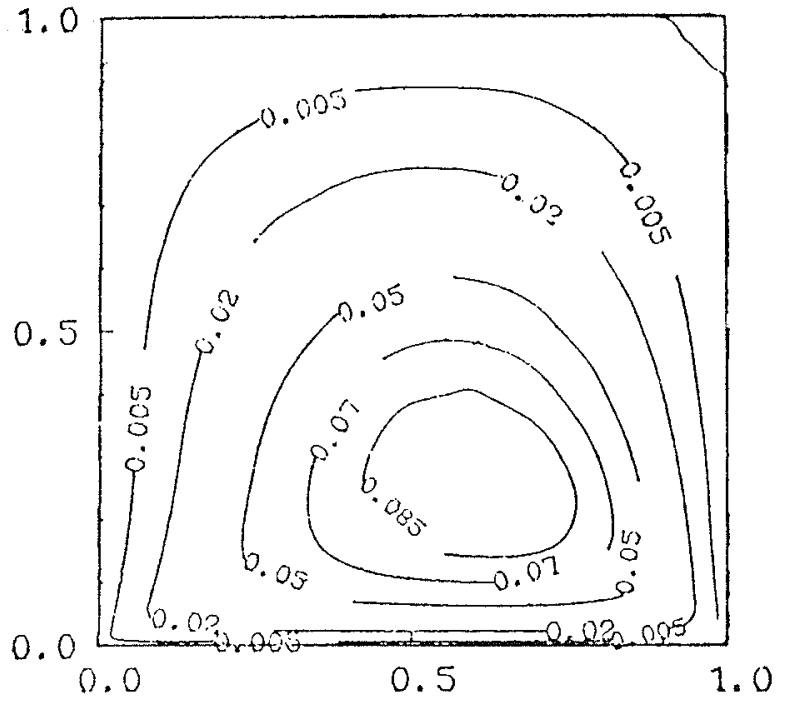

$3 A \quad G E=0$.

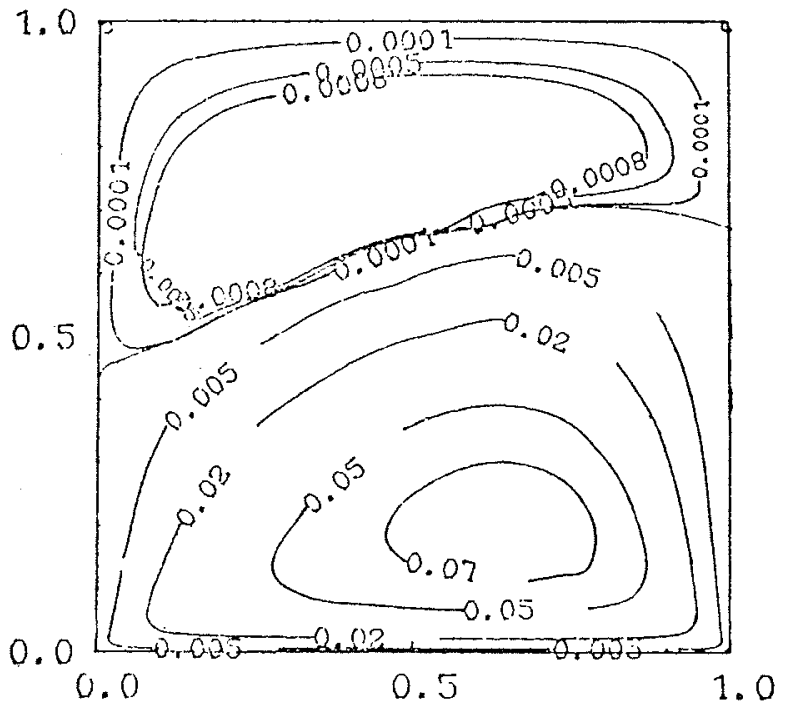

3C $G E=?$.

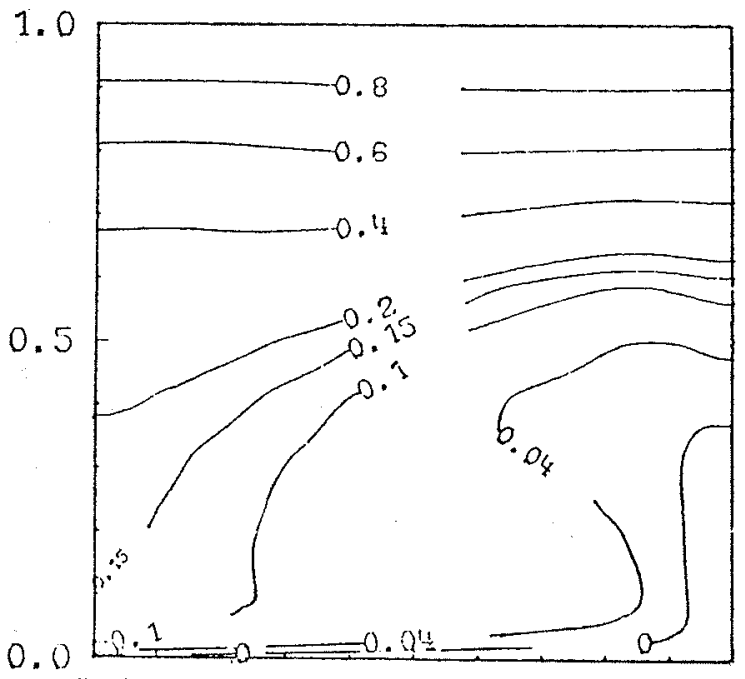
0.0
0.5
1.0

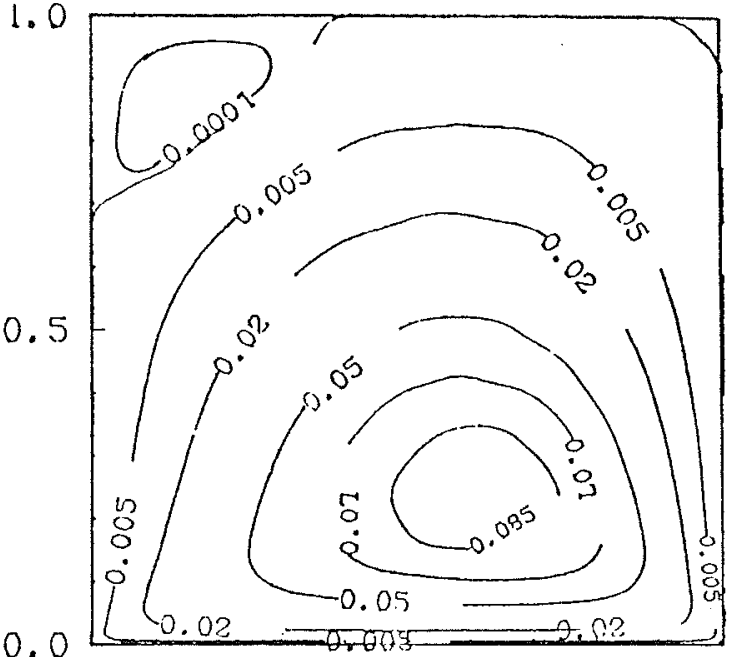

0.5

1.0

$3 B \quad G E=0.4$

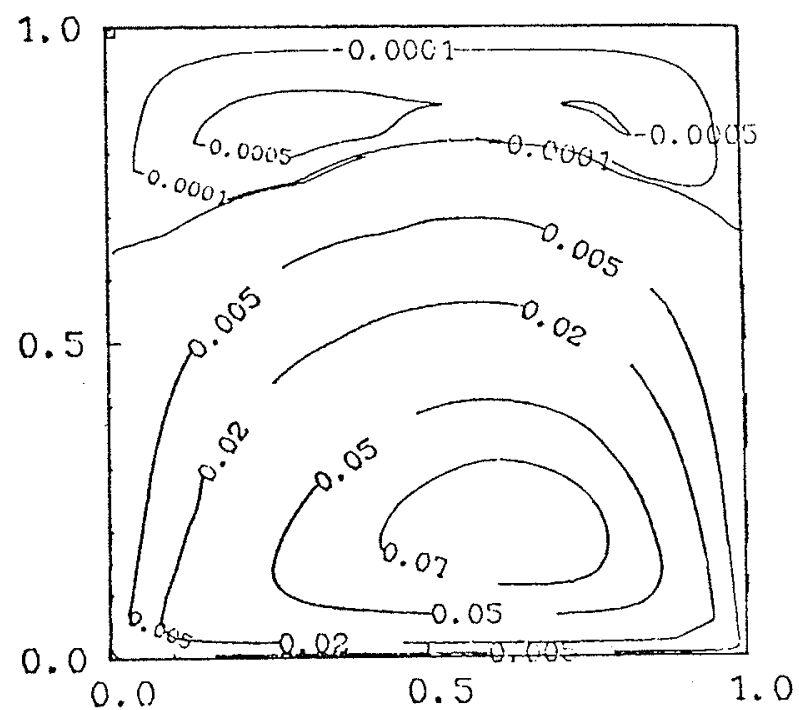

3D $G E=20 . \quad P R=0.01$

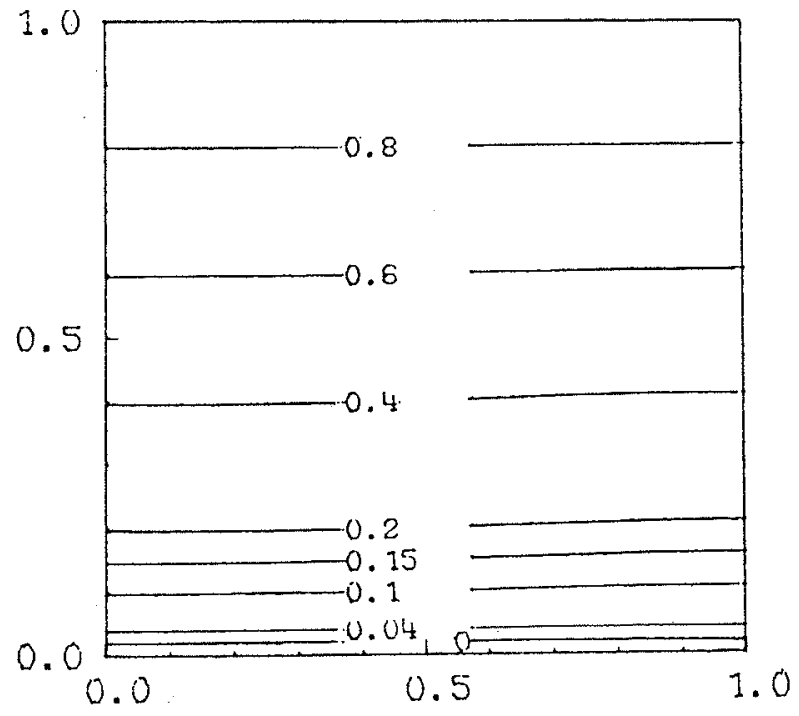

3F $\quad G E=20 . \quad P H=0.01$ 

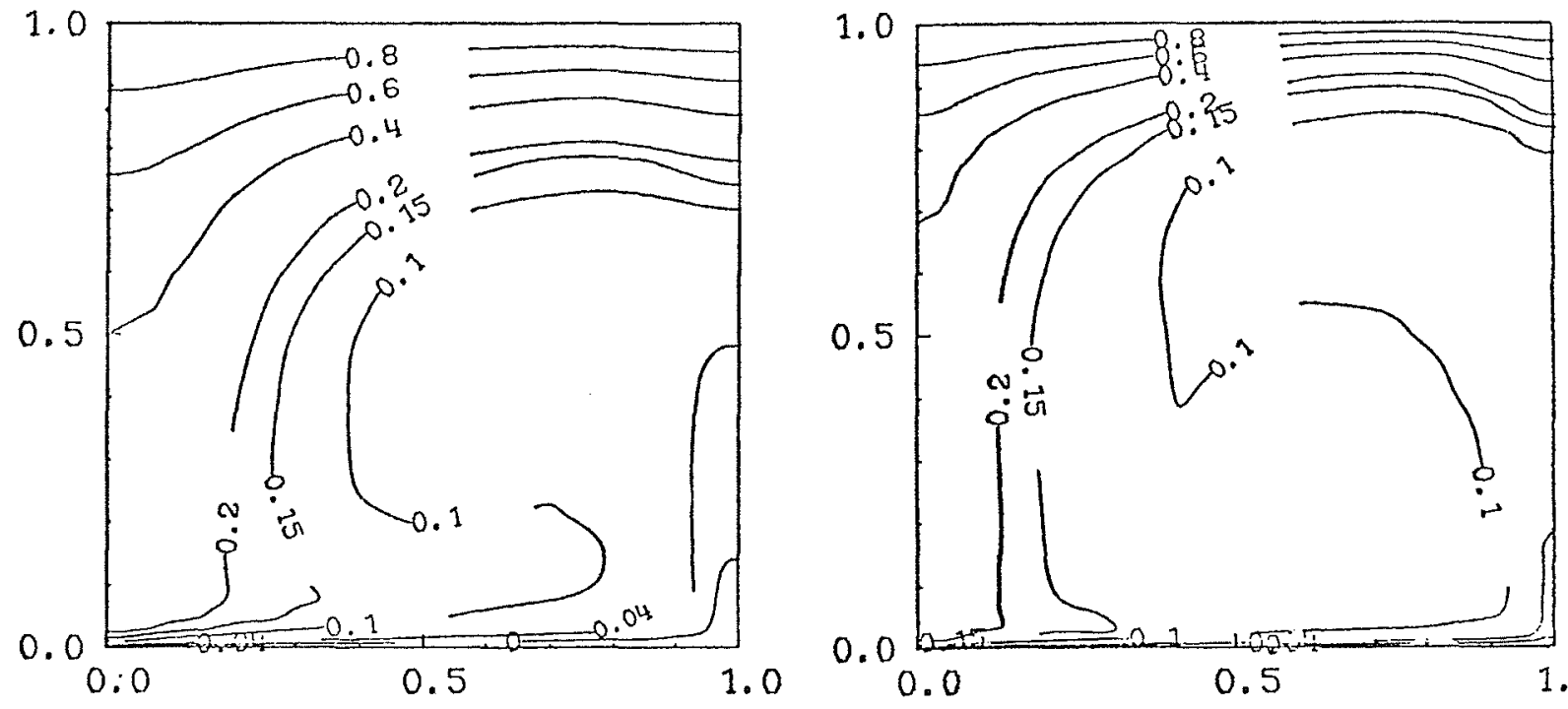

$4 \mathrm{~A} R E=100$

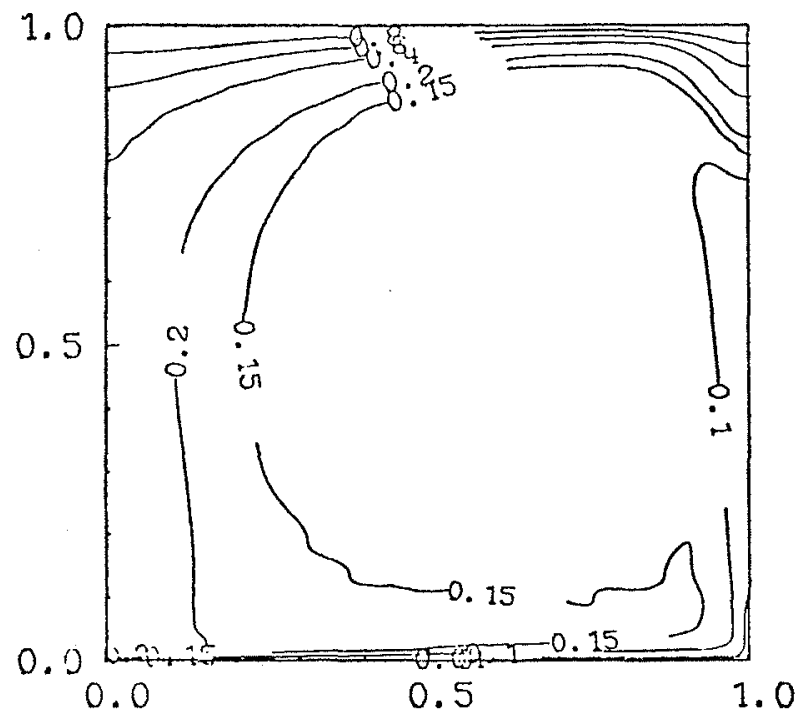

$4 \mathrm{C} R E=1500$

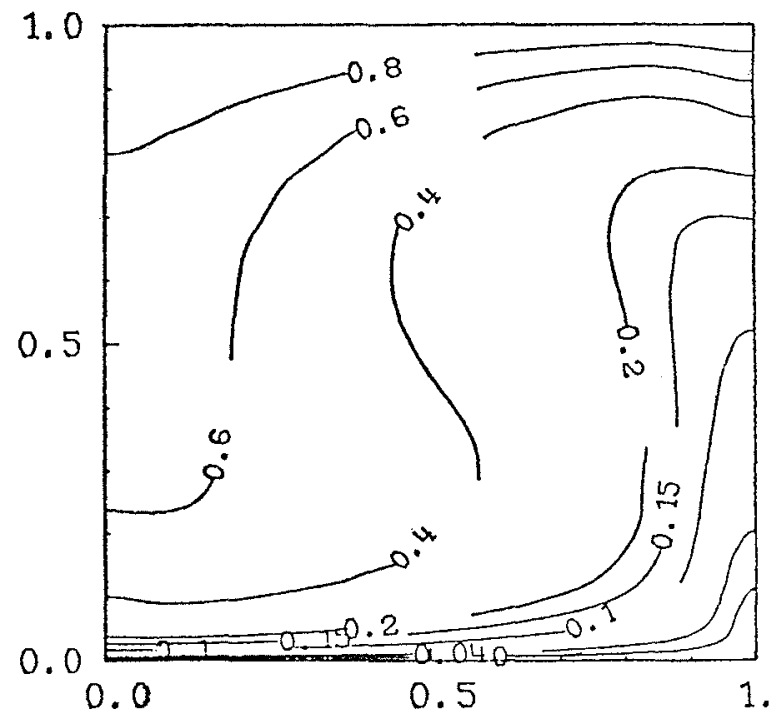

$40 \quad R E=35000 \quad P R=0.01$

4/ $\mathrm{GE}=0$. 
dispose d'un support transparent formé de bandes colorées parallèles. En l'absence de gradient thermique dans la section d'essais, le faisceau traverse la bande centrale. $\mathrm{Si}$ localement les rayons lumineux rencontrent un gradient d'indice, ils sont déviés et traversent une autre bande colorée; sur l'image, dans le plan d'observation de la section d'essais, la zone perturbée apparaît alors avec une couleur différente du reste de l'écoulement.

Les deux moyens d'investigation: mesures locales de température par thermocouples, visualisation par strioscopie bien qu'apportant des résultats en accord qualitatif, offrent le maximum d'informations dans des domaines expérimentaux différents.

Pour la strioscopie, deux limites restreignent l'emploi de la méthode:

- si les gradients thermiques sont trop importants dans l'ensemble de l'écoulement, l'image peut être considérablement déformée;

- si l'écoulement est fortement turbulent, sa complexité rend difficile l'interprétation des clichés.

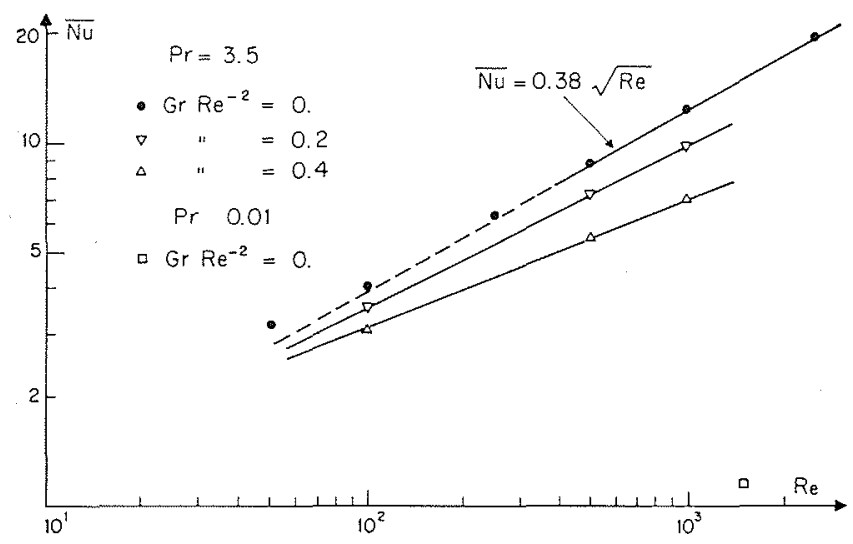

5/ Nusselt moyen.

\section{2 - Résultats}

Cette série d'essais a couvert le domaine expérimental suivant :

- pour le nombre de Reynolds calculé sur la vitesse moyenne de l'écoulement en canal:

$10^{3}$ à $6 \cdot 10^{4}$

- la densité de flux thermique imposé à la face supérieure de la cavité :

$$
0,05 \text { à } 9,5 \mathrm{~W} / \mathrm{cm}^{2}
$$

- soit pour le paramètre qui caractérise les échanges par convection mixte, $\mathrm{Gr} * / \mathrm{Re}^{2}\left(\mathrm{Gr}^{*}\right.$ étant le nombre de Grashof modifié égal à $\overline{\mathrm{Nu}}$. Gr)

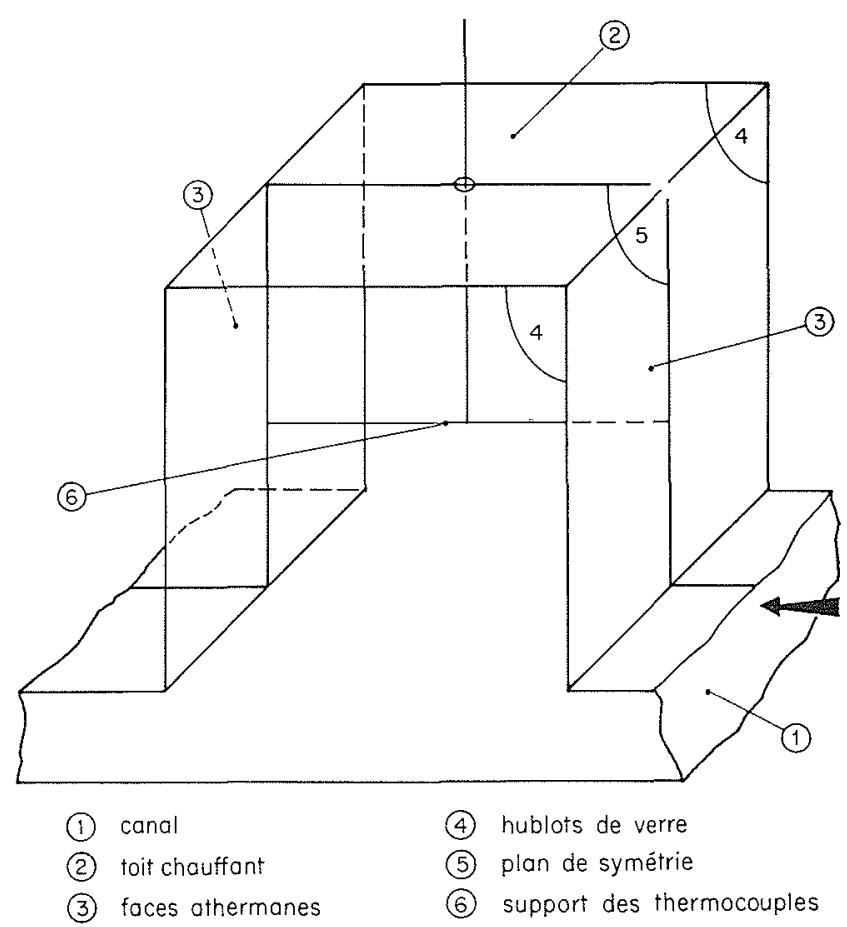

6/ Dispositif expérimental.

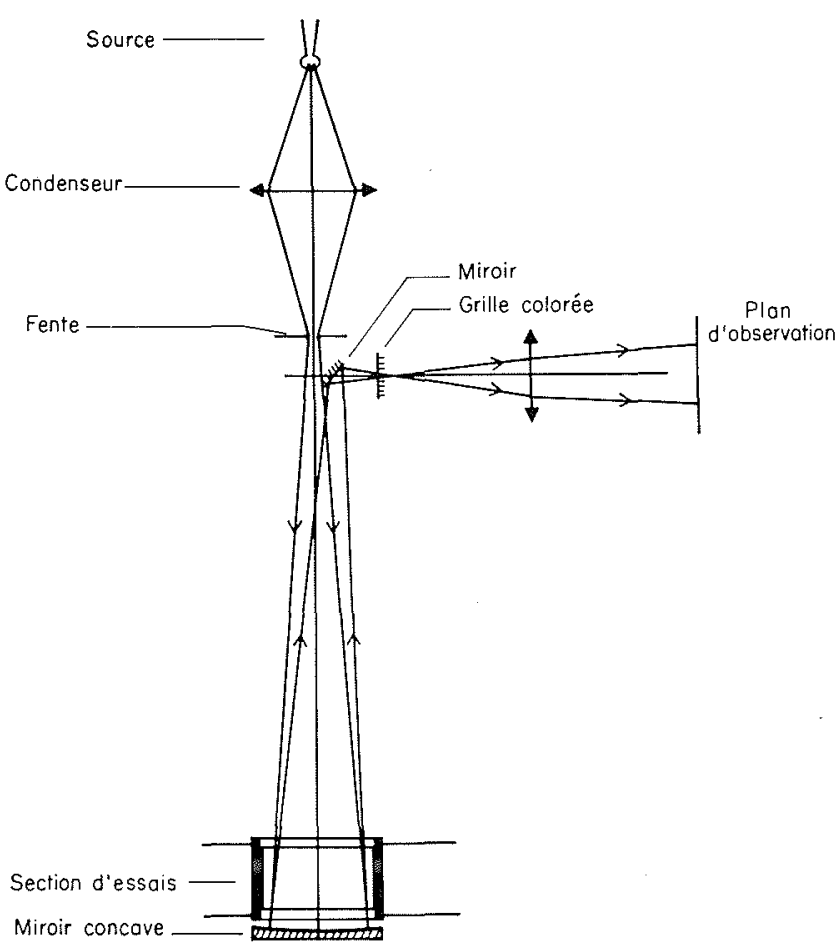

7/ Montage optique. 
Le calcul des nombres adimensionnels est effectué en prenant les propriétés physiques à la température de l'eau d'entrée du canal; la dimension caractéristique retenue est la largeur de la cavité.

Trois essais sont présentés dans la planche couleur (fig. 8):

A) Le premier (fig. 8 A) correspond à une valeur du nombre de Reynolds de 1 100, soit la plus faible présentée ici.

Dans la partie supérieure de la cavité, la présence d'une plage de couleur unie révèle l'existence d'une zone de gradient constant. La valeur de ce gradient vertical peut être estimée comprise entre 1 et $2,1^{\circ} \mathrm{C} / \mathrm{cm}$. De même, la teinte de l'écoulement en canal indique que la température y est uniforme. Comprise entre ces deux zones, apparaît la perturbation de la stratification par la convection forcée. Elle comprend:

- la ligne de séparation entre l'écoulement en canal et l'écoulement dans la cavité; celle-ci apparaît nettement de par l'existence d'un gradient vertical localisé à cet endroit;

- une zone de recirculation qui est centrée vers l'aval de la cavité; la teinte qui lui correspond marque l'existence d'un gradient vertical de température; sa valeur est inférieure à celle enregistrée dans la zone soumise à la stratification stable.

Dans cet essai l'écoulement est laminaire.

B) Pour une valeur supérieure du nombre de Reynolds ( $R e=4000$ - fig. 8 B) la recirculation occupe une zone plus importante de la cavité. Toutefois, une zone de stratification stable demeure sous le toit de la cavité.

Des instabilités apparaissent au niveau de la ligne de séparation entre l'écoulement dans la cavité et celui dans le canal. Le battement de la ligne de séparation contre le coin aval engendre la formation de tourbillons qui sont convectés ensuite par le courant de recirculation. Un film réalisé sur ces essais le montre. Ainsi on peut localiser la source de la turbulence qui apparaît dans la zone de recirculation, à la couche de mélange entre celle-ci et l'écoulement en canal.

L'accroissement de la zone de recirculation avec le nombre de Reynolds, lorsque le nombre de Grashof est maintenu constant, est un résultat intéressant bien mis en évidence par la comparaison de ces deux essais. Un moyen de caractériser cet accroissement est de repérer la position du point de décollement de la couche limite qui se développe sur la face verticale aval de la cavité. L'analyse de l'ensemble des essais réalisés fait apparaître que cette position dépend uniquement du groupement adimensionnel $\mathrm{Gr}^{1 / 3} \mathrm{Re}^{-2}$. Pour des valeurs de ce groupement inférieures à $10^{-5}$, la zone de recirculation occupe l'ensemble de la cavité.

C) La dernière photographie (fig. $8 \mathrm{C}$ ) montre la visualisation d'un tel écoulement où le tourbillon principal occupe l'ensemble de la cavité. La nature fortement turbulente de l'écoulement permet difficilement d'en faire une interprétation finie. Toutefois il apparaît que le gradient vertical de température est faible dans une grande partie de l'écoulement, sauf dans la couche limite thermique qui se développe sous le toit chauffant de la cavité.

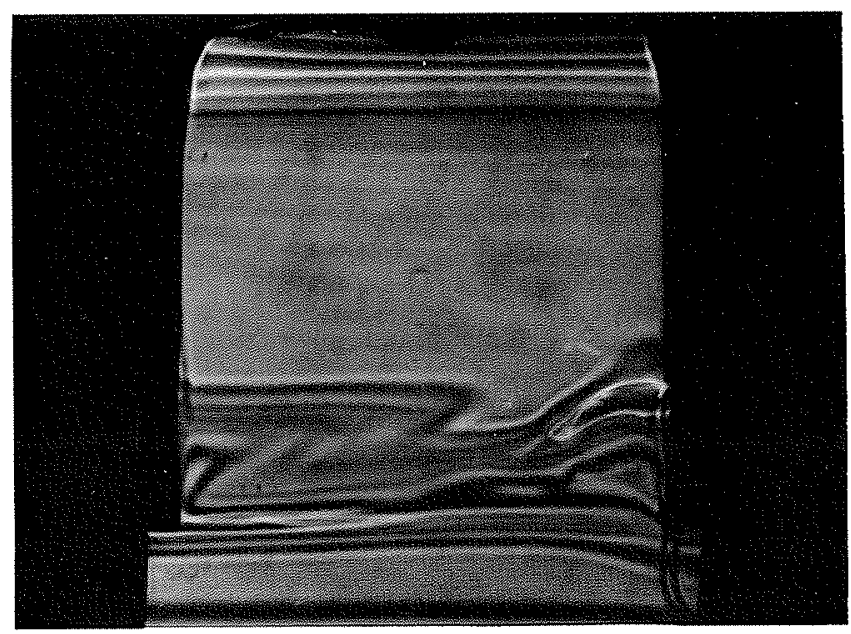

A

$\operatorname{Re}=1100$

$\mathrm{Gr} / \mathrm{Re}^{2}=80$

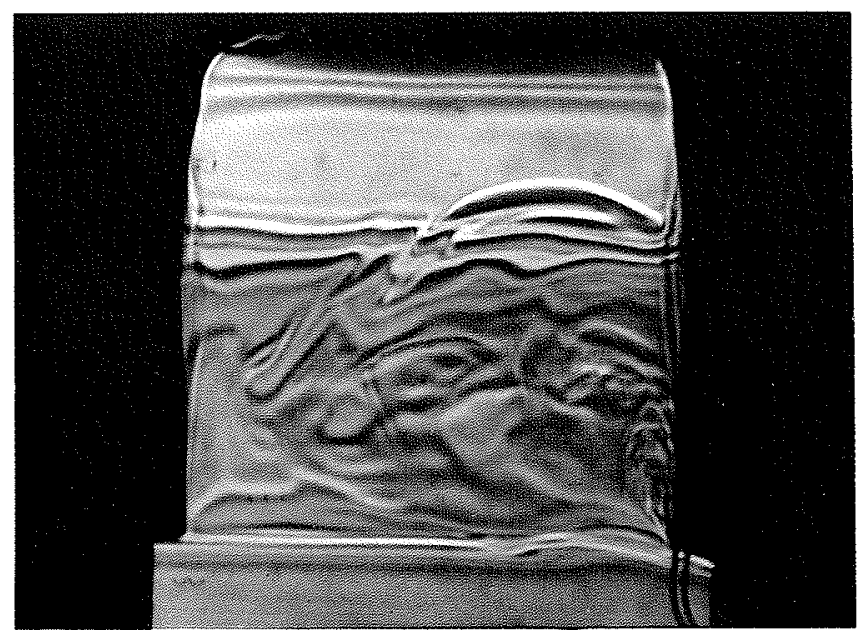

B/

$R e=4200$

$\mathrm{Gr} / \mathrm{Re}^{2}=6,5$

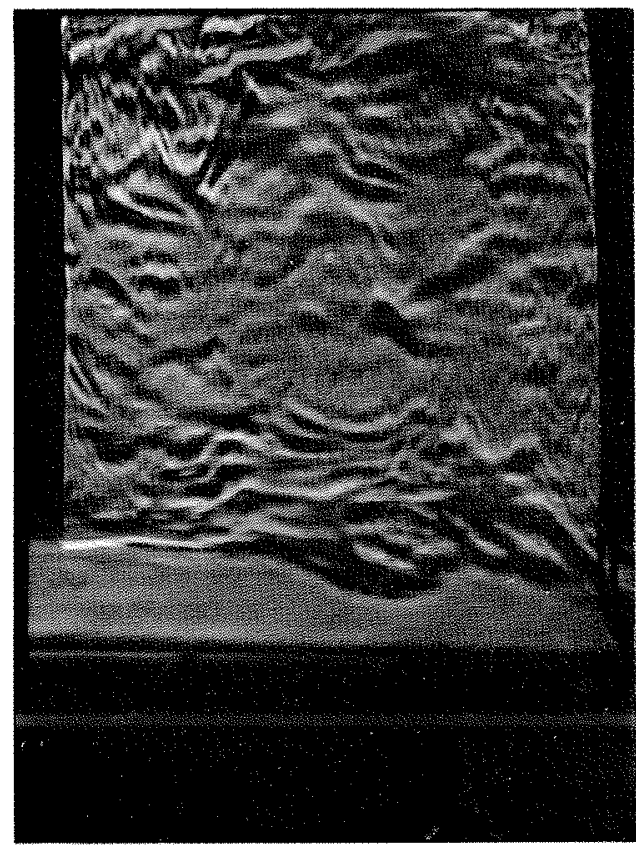

Cl $\quad \operatorname{Re}=9000$

$\mathrm{Gr} / \mathrm{Re}^{2}=6$

8) Visualisation de l'écoulement 


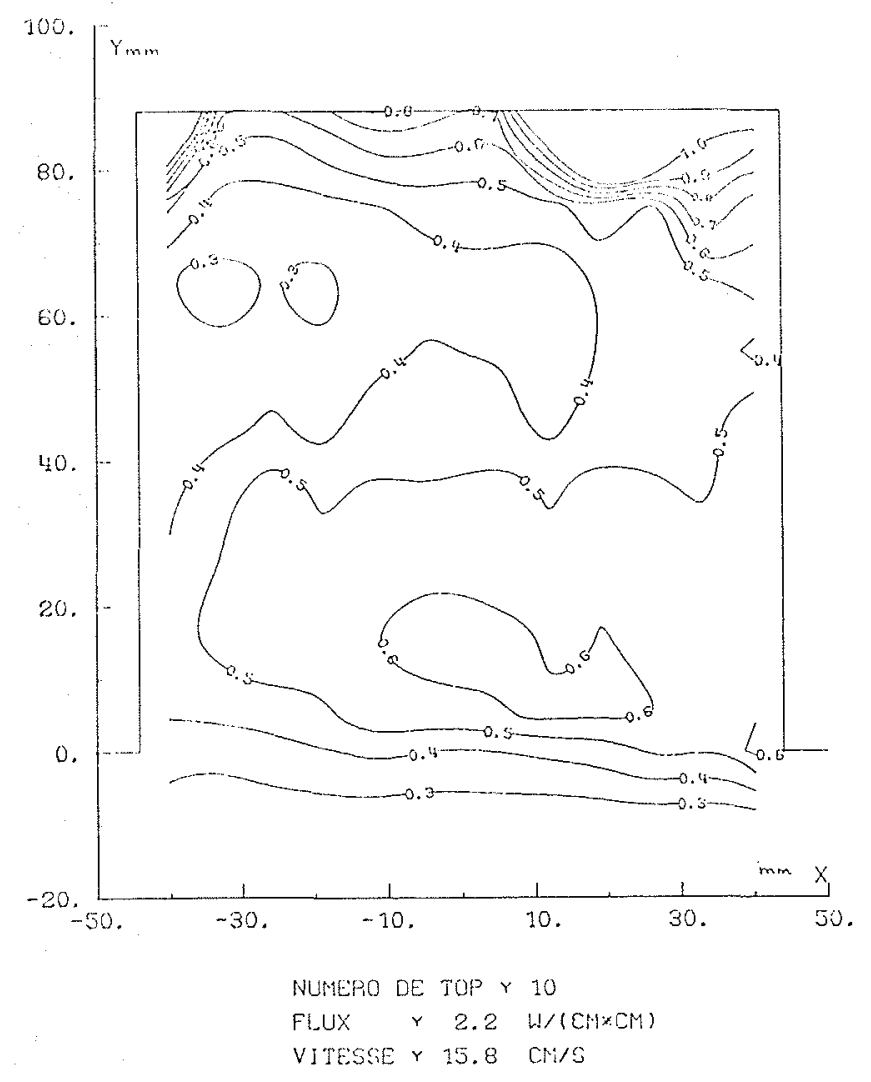

91

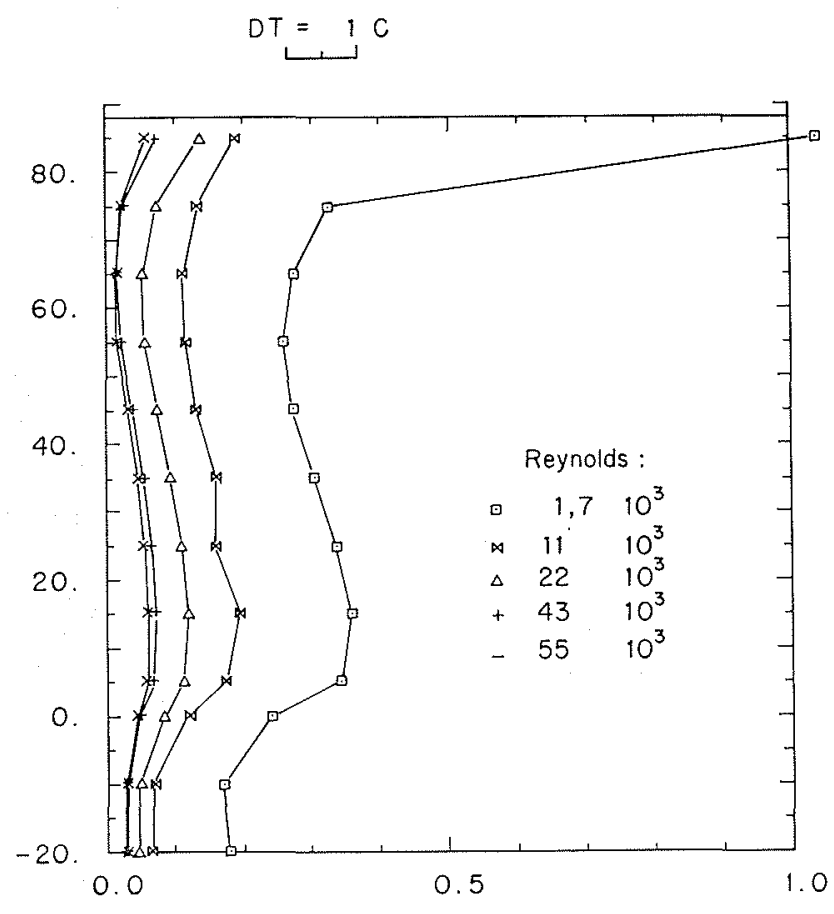

La figure 9 présente le tracé des isothermes pour une configuration d'écoulement analogue à la figure $8 \mathrm{C}$ obtenue avec des valeurs différentes des paramètres adimensionnels. On y retrouve le résultat observé sur la visualisation de l'écoulement: le resserrement des isothermes au voisinage du toit et leur espacement dans la partie centrale correspondant à une distribution presque uniforme dans le cœur de l'écoulement. Les isothermes y présentent par ailleurs une forme en $\mathrm{S}$ caractéristique. Ceci traduit une inversion du gradient thermique dans la partie centrale.

Pour compléter ces résultats, on présente dans la figure 10 les profils verticaux de température au voisinage de l'axe de la cavité pour une même valeur du nombre de Grashof, et différentes valeurs du nombre de Reynolds. L'uniformisation et la diminution de la température dans la partie centrale de la cavité y sont manifestes.

\section{4 - Conclusions}

Cette contribution à l'étude de la convection mixte dans une cavité a montré la complémentarité des aspects théoriques et expérimentaux.

Lorsque les forces de volume sont prépondérantes, une stratification stable est présente dans une grande partie de la cavité. L'effet de la recirculation est d'uniformiser localement le champ de température au voisinage du canal. Lorsque les effets convectifs sont importants, la zone occupée par la recirculation est plus grande. Dans ce cas, la température est pratiquement constante dans l'ensemble de la cavité en dehors des couches limites sur les parois. $\mathrm{Sa}$ valeur est proche de la limite froide.

Ces résultats, communs aux études théoriques de l'écoulement laminaire et aux écoulements turbulents observés expérimentalement, tendent à montrer que la turbulence a peu d'influence sur la structure des phénomènes considérés; toutefois elle intervient sur les valeurs de débit recirculé et de la quantité de chaleur échangée.

Enfin les résultats obtenus pour le cas des métaux liquides montrent que la stratification est beaucoup plus difficile à rompre puisqu'il faut atteindre des nombres de Péclet extrêmement grands pour uniformiser la température. Ce brassage thermique a d'autant plus de mal à se produire que les forces de volumes sont importantes.

Des informations intéressantes pour la thermohydraulique de l'intercuve découlent certainement de cette étude. Elles ne donnent encore que des tendances qualitatives. Des calculs numériques avec métal liquide et pour des valeurs des paramètres adimensionnels plus proches de ceux de l'intercuve sont en cours. Dans ce domaine, on a noté que les difficultés de convergence, posées par les valeurs élevées du paramètre de la convection mixte, sont atténuées par des liquides à faible nombre de Prandt1. Des études expérimentales sont poursuivies parallèlement avec un fluide classique, et un métal liquide, afin de mettre en évidence les influences de l'élancement de l'intercuve et du nombre de Prandtl. 


\section{Références}

[1] Batchelor (G. K.). - J. Fluid Mech., 1, 177 (1956).

[2] Squire (H. B.). - J. Roy. Aero. Soc., 60, 203 (1956).

[3] Grimshaw (R.). - J. Fluid Mech., 39, 695 (1969).

[4] Burggraf (O. R.). - « Proc. of the 1965 Heat Transf. and F1. Mech. Inst. », Standford Univ. Press.

[5] Gosman (A. D.) et al. - "Heat and Mass Transfer in recirculating flows ». Academic Press (1969).

[6] Mills (R. D.). - J. Roy, Aero. Soc., 69, 714 (1965).
[7] PAN et ACrivos (A.). - J. Fluid. Mech., 28, 643 (1967).

[8] Prandtl $(\boldsymbol{L}$.$) . - " Guide à travers la mécanique des fluides \%,$ Dunod (1952), 448 pages.

[9] Grand (D.), Latrobe (A.), Vernier (Ph.). - «Etude des courants de recirculation dans une cavité $*$ (2* partie: «Ecoulement non isotherme »). CEA R 4448 (2) (à paraître).

[10] Béhar (M.) et Semeria (R.). - La strioscopie appliquée à l'étude de l'ébullition et du dégazage. La Houlle Blanche 6, 687 (1963).

[11] Hauf (W.) et Grigull (U.). - Optical methods in heat Transfer Advances in heat Transfer, 6 (1970).

\section{Discussion}

Président : M. H. MONDIN

M. le Président remercie M. Grand pour son exposé et pour la présentation du remarquable film qu'il a clairement commenté.

11 ouvre ensuite la discussion en posant la question suivante

Les gradients le long des parois sont-ils plus forts dans le film (essais à l'eau) que dans l'expérience en sodium faisant l'objet de la figure $\mathrm{n}^{\circ} 3 \mathrm{~F}$.

Oui, répond $M$. Grand, la distribution et l'évolution du gradient sont différentes : au lieu d'une évolution linéaire, on observe un abaissement de température à la partie basse avec un raccordement sous le toit par un gradient plus élevé.

Quelles sont les conclusions les plus nettes tirées de ces expériences pour ce qui concerne les problèmes posés par «l'intercuve» de Phénix ? demande M. le Président.

M. Grand répond :

Ce type de modélisation montre que l'effet des jets, à la sortie des échangeurs, devrait être assez important sur la distribution des températures en partie haute; il en résulte une certaine homogénéisation, d'où refroidissement du sodium au-dessus de la sortie de l'échangeur et report du gradient sous le toit de l'intercuve.

Ces effets sont beaucoup plus significatifs sur les résultats obtenus «en eau» qu' en sodium » car, en sodium, en raison de la valeur du nombre de Prandtl, la stratification est beaucoup plus diflicilement détruite par la convection. Au vu de ces résultats, la distribution des températures dans l'intercuve devrait se situer entre une distribution linéaire, correspondant à la conduction, et un léger amortissement de la distribution linéaire en partie basse, laquelle se raccorde avec les parties supérieures de l'intercuve par un gradient un peu plus important que celui observé en conduction pure.

Il est vrai que, puisque dans nos expériences le jet était relativement guidé par un canal horizontal, lentraînement qu'il peut provoquer - donc la recirculation qu'il peut induire - est beaucoup plus fort que si les jets des échangeurs sortaient en partie basse.

M. MEjane (C.E.A.) souhaiterait une comparaison entre l'expérience et les résultats des calculs exposés dans la communication, en particulier du fait que la condition aux limites imposée dans ces derniers n'est pas tout à fait la même que celle observée dans l'expérience (battements au niveau de l'interface entre la circulation générale dans le canal et celle dans la cavité).

M. Grand donne les précisions ci-après :

La comparaison de l'évolution montrée dans le film et de celle résultant du calcul numérique, donne deux résultats d'ordre qualitatif concernant l'interaction entre la circulation et la stratification : - d'une part, le refroidissement de la zone de recirculation lorsque

la vitesse augmente;
- d'autre part, le tassement, la destruction progressive de la stratification par une zone de recirculation qui croît avec la vitesse dans le canal.

Effectivement, les conditions aux limites dynamiques sont diffé rentes dans les deux approches; mais celles-ci n'interviennent qu'au second ordre dans le problème qui nous intéresse.

Dans le très bon film que nous avons vu, demande M. CoAntrc, quelles étaient les conditions de l'écoulement dans le canal ? S'agissait-il d'un écoulement qui avait été délibérément mis dans l'état laminaire ou d'un écoulement turbulent?

L'écoulement était établi entre deux plaques; je pense quil était laminaire et nous n'avons pas cherché délibérément à le rendre turbulent, répond $M$. Grand.

Il me semble, poursuit $M$. Coantic, quil y a me diférence assez fondamentale entre ce qui se passe dans l'hypothèse d'un écoulement laminaire et dans le cas d'un mouvement turbulent.

Dans la réalité, l'écoulement à la base de la cavité sera généralement turbulent. Dans un modèle mathématique avec écoulement laminaire, la condition de conservation de la masse implique éviciemment que la surface séparant la cavité de l'écoulement extérieur soit imperméable à la masse; mais, en réalité, il y a un échange de quantité de mouvement et un échange de chaleur par échange de particules fluides, très visible dans le film. Il en résulte que, pour un nombre de Reynolds donné, la mise en circulation à l'intérieur de la cavité va être beaucoup plus efficace et la quantité de chaleur emportée de la cavité beaucoup plus grande.

Je pense que les méthodes que vous emplovez sont plus ou moins issues des travaux de Spalding et Patankar. N'existe-t-il pas une version de ces méthodes de calcul permettant de tenir compte de la turbulence au moins de façon empirique?

Nous avons procédé, en effet, répond $M$. Grand, à divers calculs utilisant les méthodes de Harlow, Daly, basées sur la conservation de l'énergie cinétique de la turbulence. Mais nous n'avons pas une confiance telle dans ces moyens d'approche de la turbulence pour nous permettre d'en présenter ici les résultats.

Mais la remarque que vous faites est tout à fait pertinente. Nons avons pu observer expérimentalement des entraînements turbulents de l'ordre de trois à quatre fois supéricurs à ceux qu'on pouvait calculer en régime laminaire.

M. Ie Président clôt la discussion en remerciant tous ceux qui l'ont animée et donne la parole à M. LALl.ement pour l'exposé de la communication de MM. J.-M. GAMms et J. BRANCHU, qui, à leur grand regret, n'ont pu assister à la présente réunion. 